\title{
Descortesía y comportamientos sexistas reproducidos por mujeres en la cultura rioplatense
}

\section{Impoliteness and sexist behaviors of women in the culture of Río de la Plata}

\author{
Silvia Kaul de Marlangeon \\ Universidad Nacional de Río Cuarto, Río Cuarto, Argentina \\ silviakaul@gmail.com
}

Gacceso abierto / OPEN ACCESS

Cita: Kaul de Marlangeon, Silvia (2019). Descortesía y

comportamientos sexistas reproducidos por mujeres en la cultura rioplatense. Textos en Proceso, 5(2), pp. 49-60. http://doi.org/10.17710/tep.2019.5.2 4kaul

Editors: Ariel Cordisco, Instituto de Lingüística, Universidad de Buenos Aires, Argentina; Ma Eugenia Flores Treviño, Universidad Autónoma de Nuevo León, México

Recibido: 29/11/2019

Aceptado: 02/03/2020

Conflicto de intereses: La autora ha declarado que no posee conflicto de intereses.

Copyright: (c) Silvia Kaul de Marlangeon. Esta obra está bajo licencia Creative Commons Reconocimiento 4.0

\section{Resumen}

El presente trabajo se propone analizar actividades de imagen descorteses surgidas de comportamientos verbales sexistas internalizados y recreados por las propias mujeres, comportamientos que concurren a modelar la identidad social femenina en la cultura rioplatense. Tales actividades de imagen descorteses se extraen de un corpus de discurso mediático recogido en Internet. Esta investigación se enmarca teórica y metodológicamente en la pragmática sociocultural, en el análisis del discurso de descortesía del español rioplatense. Los resultados del análisis muestran que, por un lado, tales comportamientos verbales sexistas y descorteses asumidos como propios por parte de mujeres contribuyen a promover y a propagar la ideología androcéntrica del universo de creencias del grupo del que las mujeres forman parte; $\mathrm{y}$, por el otro, aseguran la pervivencia de premisas culturales que representan estereotipos negativos de la imagen social femenina.

Palabras clave: actividades de imagen descorteses; sexismo verbal; estereotipos; premisas culturales; pragmática sociocultural

\section{Abstract \\ In this study we analyze impolite facework activities arising from sexist verbal behaviors internalized and recreated by women themselves. These behaviors contribute to shaping the feminine social identity in the culture of Rio de la Plata in Argentina. Such facework activities are extracted from a corpus of media discourse collected on the Internet. This research is framed theoretically and methodologically in the sociocultural pragmatics, and in the analysis of impoliteness discourse within Spanish variety of Rio de la Plata. The results show that, on the one hand, sexist and impolite verbal behaviors assumed as their own by women contribute to promoting and propagating the androcentric ideology of the universe of beliefs of}


these women's group; on the other, they ensure the survival of cultural premises that represent negative stereotypes of the female social face.

Keywords: impolite facework activities; verbal sexism; stereotypes; cultural premises; sociocultural pragmatics

\section{Introducción}

Los comportamientos sexistas han sido estudiados desde diversos ámbitos, sobre todo desde la Sociología (Lipovetsky, 1997) o desde la Psicología social (Glick y Fiske, 1999).

Lipovetsky (1997, passim) analiza tres modelos de mujer a lo largo de la historia. La primera mujer se prolongó desde el inicio hasta los albores del siglo XIX. La segunda mujer coexistió con la primera mujer a partir del siglo XII d. C., época en que el código cortés desarrolla el culto a la bella dama amada. Lipovetsky (1997, p. 208) denomina exaltada a esta segunda mujer vigente dentro de una cultura aristocrática de celebración al bello sexo y de exaltación a la madre. Esta evoluciona hacia la sacralización de la esposa-madre-educadora durante el siglo XIX, pero sin alcanzar ninguna independencia intelectual, económica o política y siempre subordinada al hombre. En la actualidad, la tercera mujer o mujer sujeto es un modelo que conjuga discontinuidad y continuidad: roles modernos y roles antiguos cohabitan, pues aún continúan vigentes las desigualdades entre los sexos, aunque ahora en una época en que los valores machistas han sido finalmente devaluados, la mujer no tiene una identidad sólo constituida por sus funciones de madre y esposa, sino también por su profesión o trabajo y ha logrado entrar de lleno en el mundo meritocrático, es decir, basado en la inteligencia, el esfuerzo y la competitividad que hasta ahora había estado reservado como coto privado a los hombres.

Glick y Fiske (1999) estudian el sexismo ambivalente o sexismo moderno en el cual conviven dos tipos de creencias sexistas: el sexismo tradicional, pleno de sentimientos negativos hacia las mujeres (sexismo hostil) y otras formas de sexismo enmascaradas y más sutiles que consideran a las mujeres en forma estereotipada y restringidas en sus posibilidades (sexismo benevolente). En estas formas encubiertas de sexismo las mujeres son retratadas como personas bellas pero representadas como incompetentes para las tareas importantes, sobre todo, de índole económica, legal, gubernamental o política.

La combinación de estas formas de sexismo hostil y benévolo producen ambivalencia de sentimientos y comportamientos entre las personas: hay grupos de mujeres que merecen un tratamiento hostil y otras, las que aceptan la supremacía masculina, uno benevolente. Ambas ideologías sexistas son complementarias, comunes a todos los grupos humanos y culturas y funcionan para mantener las desigualdades entre hombres y mujeres.

El presente artículo analiza actividades de imagen descorteses surgidas de comportamientos verbales sexistas internalizados y recreados por las propias mujeres, comportamientos que concurren a modelar la identidad social femenina en la cultura rioplatense y a exhibir cómo estas creencias sexistas son propagadas en el seno de dicha cultura.

Se adopta el marco teórico de la Psicología Social con relación a temas tales como sexismo o ideología de género (Glick y Fiske, 1999; Moya, Expósito y Padilla, 2006), estereotipos (Fiske et al., 2002). Asimismo, se apela al marco teórico y metodológico de la Pragmática Sociocultural, lo cual implica examinar tales 
actividades de imagen descorteses inmersas en el contexto situacional y cultural de la comunidad de habla en cuestión y considerar el contexto del usuario como sostén de la interpretación (Bravo, 2009, 2010).

Las actividades de imagen descorteses que aquí se tratan se extraen de un corpus de discurso mediático recogido en Internet y relacionado con la presencia de actitudes sexistas arraigadas en el seno de la comunidad rioplatense, reproducidas por mujeres en hechos de la vida diaria y difusoras de la ideología machista.

Este trabajo continúa con la sección 2, que comunica el marco teórico y metodológico. Luego, la sección 3 se aplica a perfilar los comportamientos verbales sexistas y su relación con las premisas culturales y los estereotipos asociados. La sección 4 brinda el análisis de seis ejemplos del corpus natural recogido. La sección 5 ofrece la discusión y, por último, la sección 6 presenta las conclusiones.

\section{Marco teórico y metodológico}

La perspectiva pragmática y sociocultural del análisis del discurso de la (des)cortesía verbal (Bernal, 2007; Bravo, 2009, 2010) es el marco teórico apropiado para analizar el uso situado de los recursos comunicativos lingüísticos dentro de su propio sistema sociocultural.

También constituye el marco metodológico (Kaul de Marlangeon, 2012, 2017) que permite proceder inductivamente de modo tal que los textos del corpus son el instrumento para analizar en ellos conductas humanas expresadas por medio del lenguaje. Tales conductas se plasman en dichos textos como producto de la actividad discursiva. Las conductas se analizan e interpretan sobre la base de las trazas lingüísticas encontradas en cada uno de los textos que remiten de modo singular a los contextos de situación y de cultura, y que permiten llegar a la comprensión de aquellos comportamientos en el seno de la comunidad considerada. Como afirma Bravo (2010, p. 50), son hipótesis socioculturales del contexto del analista que se integran al aparato metodológico como premisas socioculturales del analista.

Se recurre a la descripción émica que habilita a examinar los comportamientos del individuo, no como hechos sociales externos a él, sino desde su propia perspectiva de actor social corroborada por el analista mediante la consultación espontánea a dichos actores sociales (Kaul de Marlangeon, 2012, 2017). Ello en consonancia con la aserción de Cordisco (2020, p. 12), quien subraya el papel central del investigador para la interpretación del fenómeno de la descortesía desde una perspectiva relacional por construcción social.

Para el estudio de la descortesía verbal, el trabajo apela a los aportes de Kaul de Marlangeon (2005, 2008, entre otros) y especialmente al tipo de descortesía denominado descortesía de fustigación. Para el presente trabajo se debe tener en cuenta que las dos vertientes posibles de la descortesía de fustigación: refractariedad de un individuo hacia un grupo o afiliación exacerbada de un individuo a un grupo conciernen ambas a un determinado rasgo o aspecto ideológico del respectivo grupo, negligiendo para tal propósito cualquier otro posible rasgo o aspecto ideológico común del mismo grupo. Es posible que la afiliación exacerbada de una persona a un grupo no necesariamente implique la refractariedad de dicha persona al grupo antagónico. Por ejemplo, una mujer exacerbadamente afiliada al grupo de las sexistas, puede guardar una actitud prescindente y no refractaria respecto del grupo de las no sexistas al que adhiere su hija. 
Dentro de un amplio corpus, se analizan seis ejemplos representativos de comportamientos sexistas reproducidos por mujeres que actúan como agentes difusores de la ideología machista. Tales actitudes se hallan arraigadas en el seno de la comunidad rioplatense, pues se trata de comportamientos internalizados y recreados. Los ejemplos han sido recogidos de manifestaciones mediáticas producidas en Internet.

El momento actual de advenimiento de la mujer sujeto resulta propicio para tratar la pervivencia de modelos sexistas reflejados en las relaciones cotidianas entre mujeres dentro del ámbito público, mujeres cuyos comportamientos verbales quedan expresados con descortesía y, consiguientemente, muestran hostilidad hacia sus congéneres.

La consideración de las actividades de imagen descorteses (aquí mencionadas passim) demanda además enfoques multidisciplinarios juntamente con sus conceptos teóricos para afrontar fenómenos complejos como el sexismo. En este caso, recurrimos a la Psicología social (Glick y Fiske, 1999; Moya, Expósito y Padilla, 2006) y a la Sociología (Lipovetsky, 1997) para comprobar la íntima conexión entre las manifestaciones sexistas en los discursos de mujeres rioplatenses e inferir de tales manifestaciones sus comportamientos descorteses.

\section{Comportamientos verbales sexistas. Su relación con las premisas culturales y los estereotipos asociados}

Dentro de la Psicología Social, Expósito, Moya y Glick (1998, p. 160) entienden por sexismo una actitud dirigida hacia las personas en virtud de su pertenencia a grupos basados en el sexo biológico. Se trata de una forma de discriminación que aventaja a una parte de individuos por cuestión de sexo, mientras que pone en una situación de inferioridad a otra.

Expósito, Moya y Glick (1998, p. 160-162) consideran que el sexismo tradicional u hostil y el sexismo benévolo, componentes del sexismo ambivalente, se articulan en torno de tres ideas: 1) el paternalismo dominador (las mujeres son débiles e inferiores a los hombres ) 2) la diferenciación competitiva de género (las mujeres no poseen las características necesarias para gobernar las instituciones sociales); y 3) la hostilidad heterosexual (las mujeres son peligrosas y manipuladoras de los hombres). Tanto el sexismo tradicional como el sexismo benévolo consideran a la mujer de manera estereotipada, limitada a ciertos roles sociales de esposa y madre, y a una idealización amorosa (Glick y Fiske, 1999; Lipovetsky, 1997).

Dentro de la amplitud de los estudios sobre el sexismo, este trabajo se circunscribe a los comportamientos verbales sexistas. Los estudia como conductas discriminatorias que lesionan las relaciones sociales de las personas, al estar fundadas en la supuesta inferioridad de las mujeres como grupo social. Aunque se trate de comportamientos verbales tradicional y globalmente arraigados que trascienden una cultura determinada, sostenidos tanto por hombres como por mujeres, este trabajo se limita a las manifestaciones en forma de prejuicios y creencias que se ponen de manifiesto mediante el lenguaje empleado por las mujeres en la cultura rioplatense cuando adoptan las creencias sexistas acerca de la mujer.

Este artículo analiza los estereotipos (Wolfe y Spencer, 1996; Fiske et al., 2002) que emergen de las interacciones del corpus, especialmente los estereotipos de género (Cook y Cusack, 2010), pues los comportamientos verbales sexistas reflejan la etiología societaria y reproducen estereotipos que refuerzan la percepción 
negativa de la imagen de la mujer. Sostienen Cook y Cusack (2010: 11) que el elemento clave es presumir que el grupo específico estereotipado posee ciertos atributos particulares o características específicas y que, además, cumple con esos roles. A tal punto que todas las dimensiones de la personalidad que originan que una persona sea única, por el solo hecho de incluir a dicha persona en el grupo del estereotipo atribuido, tal persona quedará filtrada por la visión generalizada que se tiene acerca de tal estereotipo.

En los casos estudiados, los rasgos estereotípicos atribuidos a la imagen femenina resultan de un consenso de opinión que identifica a ese grupo social con una evaluación negativa descortés. Tal evaluación negativa impregna la imagen femenina de características constantes que mantienen inalterable el statu quo social: un bello objeto, un ser sexy, un ser sumiso, un ser decorativo, un ser inferior, un ser doméstico, un ser irracional, un ser débil, un ser peligroso. Los estereotipos ayudan a conformar la identidad machista de los individuos y grupos que integran y dan la justificación de su comportamiento sexista.

La relación entre los comportamientos verbales sexistas exhibidos por las mujeres y las premisas culturales (Bravo, 2010), revela que tales comportamientos son aprehendidos de tales premisas culturales concernientes al grupo que integran y son el producto de la valoración positiva que tales premisas culturales otorgan a esos comportamientos. El comportamiento verbal sexista de las mujeres, objeto de este estudio, se asimila por transmisión cultural, tanto del hogar como de las convenciones sociales vigentes en la comunidad de pertenencia, a diferencia del sexismo volitivo ejercido por los hombres que responde a sus propios intereses machistas.

\section{Análisis}

1) C Delia a través de Google+ Compartido públicamente Clarin.comExtraShowFama 04/12/16 "Pico" Mónaco fue con Pampita a un evento y se cruzó con su ex, Zaira Nara:

Se le juntó el ganado

Una mujer de nombre Delia comunica la noticia publicada por Clarin.com ExtraShowFama y expresa como comentario sobre ella se le juntó el ganado, que reproduce la ideología machista que ha absorbido de su entorno social. Este comentario queda tipificado como descortesía de fustigación (Kaul de Marlangeon, 2005) a cada referente femenina ausente, pero aludida. La mujer que comete este acto se arroga poder por sobre cada miembro del colectivo femenino tratado descortésmente. Manifiesta el estereotipo de la superioridad del macho y de la subordinación de la mujer reducida a una condición animalesca (ganado), que obedece al modelo de la primera mujer en términos de Lipovetsky (1997). El ejemplo se encuadra en un tipo de sexismo hostil y, por tanto, abierto.

$\mathrm{Su}$ empleo exhibe la premisa cultural de que la sociedad argentina tiende a reconocer la condición de macho (hombre que posee en forma destacada su masculinidad) sólo a aquel varón al que se le conocen varias hembras sucesivas o simultáneas.

En cuanto a los sentimientos que envuelve la frase, la mujer parece reflejar con ella, o bien una torpe afiliación al grupo de las sexistas, o bien envidia hacia las más "afortunadas" que forman parte del ganado de un macho, sujeto valorado 
socialmente como un triunfador, un conquistador, un seductor y la mujer un ser inferior, condenada a la dependencia del macho.

2)@AmelieGranata

El Cancer de mama es la 1 er causa de muerte de mujeres en Argentina, no las veo con el peñuelo verde pidiéndole al Estado prevención y tomografos para las mas vulnerables \#coherencia \#CuidemosLasDosVidas

La crítica de la modelo-periodista-política, Amalia Granata, al grupo de mujeres de pañuelo verde (las partidarias de la legalización del aborto y de su gratuidad en la Argentina) ilustra un caso de descortesía de fustigación a ese grupo de mujeres que le resulta antagónico, crítica agravada por esgrimir esa descortesía en un momento inoportuno, aprovecharse de la noticia de la muerte por cáncer de mama de una joven mujer, hija de una de las "Trillizas de Oro" cantantes y presentadoras de televisión y que pertenecen al grupo opuesto al de los pañuelos verdes, que está a favor de las dos vidas.

Esa crítica fue rápidamente replicada en las redes con comentarios que contraatacaron con descortesía de fustigación para canalizar el disgusto que Granata provocó con su tweet; muchos de ellos le atribuyeron a Granata una motivación subalterna de figuración como en la opinión de Rocío, una comentadora de las redes sociales:

Que mina tan perversa. Sus ganas de figurar son mas grandes que el respeto que le debe a una madre que esta enterrando a su hija. Y despues se hace la madre de america pro-vida. Por favor!! Lo unico que la mueve a esta tipeja es el interes personal de figurar.

El siguiente comentario al tweet de Amalia Granata también es un ejemplo de descortesía de fustigación hacia su autora. Reproduce una opinión sexista internalizada por Verónica que se convierte así en una mujer afianzadora y propagadora de la ideología sexista en el seno de la sociedad rioplatense:

Veronica Sh...

Curriculum de GranGata

Acostarse con Robi Williams

Mear una maceta en GH

Convertirse en botinera

Convertirse en madre botinera

Peasearse años por los programas de chimentos reclamando cuota alimentaria

Ser "putón" en programa de Petinato

Embarazarse de empresario pizzero

Ser GranGata candidata por el frente renovador ... Perdió como en la guerra ...

Hacer cursillo de periodismo de un año de duración

El texto está poblado de estereotipos sexistas: puta, putón, mujer botinera (mujer relacionada con un jugador de fútbol), madre botinera, gata (mujer sexualmente atractiva y provocadora). Ellos son responsables de la proyección en el imaginario social de la imagen infravalorada de la mujer, encarnada en Granata que representa el estereotipo de la mujer frívola, bella, interesada. Asimismo, se advierte en el comentario de Verónica que sus emisiones están cargadas de las emociones negativas comunes a todas esas actitudes sexistas (Kaul de Marlangeon, 2017). Cuando, contrario sensu, muchas de esas mismas mujeres al referirse a 
actitudes simétricas por parte del varón, emplean epítetos atenuadores de negatividad como mujeriego o picaflor.

La biografía objetiva de Amalia Granata que aparece en Wikipedia expone:

Nació en Rosario, Santa Fe, Argentina. Es modelo y panelista, más conocida por su rol mediático. Estuvo en pareja con el exfutbolista Cristian Fabbiani, con quien tuvo una hija y luego estuvo en pareja con el empresario Leandro Squarzon, con quien tuvo un hijo. Comenzó su carrera televisiva de forma mediática, ya que confesó en un programa un encuentro íntimo con el cantante Robbie Williams cuando estaba de gira por Argentina en 2004. Tomó parte del reality "Gran Hermano Famosos" y participó del reality show "Bailando por un sueño". En 2009 fue tapa de la revista Playboy y actualmente se encuentra trabajando en política.

3) "AVISALE A YANINITA LATORRE QUE NO SUPONGA QUE YO ESTOY ENAJENADA, MENOS POR UN PROGRAMA DE TELEVISIÓN. TENGO CABEZA. NO SOY RUBIA"

dijo Moria (Casán, vedette y presentadora de televisión) a Ángel de Brito (periodista) por audio de WhatsApp.

Respuesta de Yanina (Latorre, periodista):

"SI SOS TAN FEMINISTA, DECIRME RUBIA Y HUECA... YO PUEDO PENSAR QUE VOS ESTÁS GRANDE Y GAGÁ. TE MANDO UN BESO”.

Ciudad Magazine, 18/03/18E

Mediante este audio, Moria Casán exterioriza como sexismo hostil su actitud machista internalizada al vapulear a Yanina Latorre con el estereotipo de rubia hueca, que la encasilla como mujer de poca inteligencia y sentido común. El tipo de cliché descortés rubia hueca asegura a Moria el éxito de la ofensa, es decir, su descortesía de fustigación hacia Yanina. El contraataque de esta última apela al mismo tipo de descortesía y a análogos estereotipos negativos de vejez y demencia.

4) Una reina criticó la marcha \#8M (Paro Internacional de Mujeres contra la violencia machista)

Estallaron las redes sociales.

$09 / 03 / 18$

Giuliana@GiuliFrare:

De verdad piensan que así ganan un mejor lugar en la sociedad? Somos muchas las que no nos identificamos con ese grupo. Feliz día a las que sí son ejemplos de vida!! 8:03 PM - Mar 8, 2018

Viqqi_Mercado@nomeatosigueis:

Vestida de Reina en el siglo XXI y respondiendo a la cosificación de la mujer en tu rol... 9:32 PM - Mar 8, 2018

Giuliana representa como "Reina" de la Vendimia al grupo de mujeres que prioriza la belleza física y la juventud, y expone a la mujer como objeto. Ella tiene asimilada la idea sexista de que las mujeres deben ser valoradas por su apariencia física, sin importar su inteligencia o su educación y la agrava con su mensaje de tweet, al hacer alarde de un comportamiento verbal sexista. Este es un ejemplo de sexismo ambivalente que reproduce la subordinación tradicional de la mujer: por una parte, la autora aplica para sí las ideas del sexismo benevolente al incluirse en 
el grupo de mujeres que exaltan el culto a la belleza, con lo cual fomenta su situación de inferioridad de género y, por otra parte, exhibe un sexismo hostil hacia el grupo de mujeres contrarias a la violencia machista.

Por lo contrario, la réplica irónica al tweet de Giuliana ilustra el caso de concienciación de la mujer sujeto actual (Lipovestsky, 1997).

5) Finalmente Cinthia Fernández (modelo y actriz) se reencontró con su marido en Carlos Paz. Apareció una foto que los muestra abrazados y surgieron rumores de reconciliación.

"Fue difícil, es todavía difícil verlo para mí. No quiero llorar...", admitió angustiada.

"Cuando se fue, porque ya no está en Córdoba, fue duro. Aunque no parezca, soy muy sumisa para las relaciones. Así como tengo fuerza de carácter para otras cosas, con él no lo soy”.

Fuente: pronto.com.ar, 09/02/2018

Cinthia representa la actitud machista de sumisión femenina y de persona no racional, sino decisivamente emocional. Al confesar que no puede valerse emocionalmente, encarna el "No puedo vivir sin él", propio de la cultura machista y representa la pervivencia de los ideales de la segunda mujer, en el sentido de Lipovetsky (1997), en la cultura rioplatense.

6) "Me conmueve Lamothe, me pasan cosas en el cuerpo".

El actor no se quedó atrás:

"No hay nada más lindo que el deseo. Nunca me dijeron algo así. Vos sos muy linda", le respondió, galante.

Fuente: Ciudad Magazine, 26/02/20.

Esta noticia refleja un cambio de actitud hacia el nuevo lugar de las mujeres y sus relaciones con los hombres. Hasta lo presente, las mujeres se hallaban sometidas a una moral severa y la iniciativa amorosa siempre quedaba reservada a la decisión del hombre. Contrariamente, la periodista muestra a una mujer con libre gobierno individual, análogo al del universo masculino. Para las mujeres aún sujetas a los dictámenes anteriores, se trata de una actitud reprochable. Pero no sólo se advierte en el ejemplo las relaciones entre hombres y mujeres reactualizadas con nuevos rasgos, sino que también constituye un ejemplo de extimidad o intimidad expuesta (Kaul de Marlangeon, 2018), que se comprueba como una práctica mediática significativa actual. La periodista Manguel no sólo expone desinhibida aspectos de su intimidad al actor con el propósito de seducirlo, sino a toda la audiencia. Asimismo, en correspondencia con el fenómeno de la extimidad, se constata una dilución creciente entre las esferas pública y privada por parte de todos los actores.

Comentarios:

(7) Elizabeth S...

ROMMINA MANGEL... UNA KALENTONA MAL.... REGALADA, ..... ASKEA UNA TIPA TAN P AL AIRE 
Silvina G.

@alfonsil50

Uh, me da vergüenza ajena

Soy la \#404

@LaVascaHija

El otro día al enano Kici, ahora Lamothe, ambos en pareja. Conclusión, le gusta lo ajeno, no tiene dignidad, redacta muy mal, es una veterana alzada...

En los tres comentarios que preceden se advierten actividades de imagen de descortesía de fustigación hacia Romina Manguel por afiliación exacerbada de las tres comentaristas al grupo de las mujeres tradicionales, ancladas en códigos atávicos.

Para ello tales comentaristas recurren al estereotipo de la mujer objeto que se presta fácilmente a tener relaciones sexuales con un hombre (kalentona mal...regalada, puta, veterana alzada); estereotipos aún anclados en las premisas culturales rioplatenses que consideran a la mujer predispuesta por naturaleza a las pasiones.

Asimismo, este ejemplo responde a la actitud machista adoptada por mujeres de considerar puta a la mujer que decide vivir libremente su sexualidad y, por lo contrario, atemperar la actitud simétrica del varón. También representa un caso de sexismo hostil.

El siguiente comentario ilustra la ocurrencia de una mujer que asume una postura acorde con los tiempos actuales y la instalación de nuevas premisas culturales como el respeto de la libertad sexual del individuo.

(8) Eli...

Ahora... Todos se lo toman a joda (no se lo toman en serio), si hubiese sido al revés, estarían rasgándose las vestiduras, tipo: "viste lo que le dijo ese hombre? Es un machirulo esqueroso, etc etc...". A dejar la hipocresía y falsas ideologías de lado.

\section{Discusión}

En 2. se explicó que las dos vertientes posibles de la descortesía de fustigación: refractariedad de un individuo hacia un grupo o afiliación exacerbada de un individuo a un grupo conciernen ambas a un determinado rasgo o aspecto ideológico del respectivo grupo de los varios que pueda tener en común tal grupo.

En particular, la explicación precedente se aplica a los casos de refractariedad de una mujer al grupo de las mujeres sexistas de la comunidad rioplatense y a la afiliación exacerbada de otra mujer a dicho grupo. Esta última persona no necesariamente es refractaria al grupo de las mujeres no sexistas, pues en otros aspectos ideológicos, como el gusto por una determinada moda, todas esas mujeres pueden coincidir perfectamente.

En todos los ejemplos del corpus se observa que los comportamientos femeninos analizados exhiben una actitud machista internalizada expresada como sexismo hostil hacia la(s) mujer(es) de pensamiento antagónico, a la(s) que considera(n) como enemiga(s). Tales comportamientos sexistas se vehiculizan, 
generalmente con un ataque (ejemplos 1, 2, 3 y 4) hacia el grupo de mujeres no sexistas, a causa de la desazón y el temor que este grupo le(s) provoca y la(s) hace sentir agredida(s). En los ejemplos considerados, el grupo de las mujeres no sexistas responde con un contraataque (ejemplos, 2, 3, 4 y el cuarto comentario del ejemplo 6 ), por medio de descortesía de fustigación por refractariedad a ese grupo de mujeres tradicionales.

La otra motivación de la descortesía de fustigación, que es la afiliación exacerbada al grupo sexista, viene acompañada por la defensa de los valores tradicionales del grupo sexista, pues ello les da seguridad dentro del statu quo imperante (el comentario de Verónica en el ejemplo 2 y los tres primeros comentarios del texto 6).

Las mujeres estudiadas constituyen una comunidad de práctica descortés cuyos miembros son descorteses en forma bilateral o bidireccional, hacia el grupo antagónico de mujeres que no participan de su cosmovisión y, viceversa por parte del otro grupo. El sexismo internalizado del grupo de mujeres tradicionales se muestra dado a la réplica de la descortesía, y para sus miembros, el compromiso mutuo de la. cortesía se transmuta en prevención mutua o conciencia acerca de la posible hostilidad que cada miembro puede desplegar en pos del logro de sus objetivos o de la prevalencia de su manera de interpretar el mundo. Por ello, los agravios pueden ser las formas de participación escogidas en una suerte de empresa conjunta, cuyo repertorio compartido son los modos de producir descortesía: palabras, símbolos, gestos, acciones y premisas culturales involucradas (Kaul de Marlangeon, 2014, p. 14).

Es evidente que las mujeres que tienen conductas sexistas internalizadas se identifican con su grupo y categorizan a las otras mujeres de actitudes no sexistas como un grupo contrario, es decir, son conscientes de la afiliación exacerbada a su propio grupo. Este es, precisamente, el momento en que aparecen los prejuicios y los estereotipos en las interacciones. Los estereotipos sexistas empleados en los ejemplos reflejan una imagen desvalorizada del sexo femenino de la agredida, por medio de la atribución de determinadas conductas negativas, de antemano transmitidas como una construcción cultural. En suma, estos rasgos concurren con un consenso de opinión que identifica al grupo social femenino con una evaluación negativa descortés que le asigna como característica constante y que sirve para mantener el estado de cosas social. Los estereotipos también coadyuvan a conformar la identidad machista de los individuos y de los grupos que integran, pues unos y otros encuentran en ellos la explicación y la justificación de su comportamiento sexista.

Lipovetsky (1997, p. 271) expone que la época en la que se relegaba a la mujer al espacio doméstico y se la apartaba de la sociedad política está definitivamente superada, aunque los tiempos presentes de cambio no significan de modo alguno que la equiparación de los dos sexos se haya establecido culturalmente, pues los hombres no han perdido la posición privilegiada de que gozan y que mantiene su poder. Ello es lo que contribuyen a afianzar precisamente los comportamientos sexistas de las mujeres en la cultura rioplatense.

\section{Conclusiones}

Los resultados de la descripción émica revelan que tales comportamientos incrementan la reproducción de la ideología androcéntrica del universo de creencias del grupo, cuya pervivencia se beneficia del apoyo de las propias mujeres. En tales 
comportamientos se sostiene una representación desvalorizada y cosificada de la mujer que perpetúa las asimetrías de poder existentes en las relaciones sociales y afianza las premisas culturales sexistas.

Tales premisas impulsan los comportamientos agresivos acompañados de emociones negativas que fijan el patrón conductual asumido por la comunidad y confirman que hasta la actualidad no se ha instaurado un modelo de equidad entre los sexos.

\section{Referencias}

1. Bernal, M. (2007). Categorización sociopragmática de la cortesía y de la descortesía. Un estudio de la conversación coloquial española. Estocolmo: Sotckholm University.

2. Bravo, D. (2009). Pragmática, sociopragmática y pragmática sociocultural del discurso de la cortesía. Una introducción. En Bravo, D., Hernández Flores, N. y Cordisco, A. (Eds.), Aportes pragmáticos, sociopragmáticos y socioculturales a los estudios de la cortesía en español (Vol. 2, pp. 31-68). Buenos Aires: Programa EDICE-Dunken.

3. Bravo, D. (2010). Pragmática sociocultural. La configuración de la imagen social como premisa-socio cultural para la interpretación de las actividades de verbales y no verbales de imagen. En Orletti, F. y Mariottini, L. (Eds.), La descortesía en español: ámbitos teóricos y metodológicos de estudio (pp. 19-46). Roma: Università degli Studi Roma Tre-Programa EDICE.

4. Cook, R. J. y Cusack, S. (2010). Gender Stereotyping: Transnational Legal Perspectives, University of Pennsylvania Press.

https://doi.org/10.9783/9780812205923

5. Cordisco, A. (2020). Estrategias de descortesía modalizadas deónticamente en inglés en ambientes laborales argentinos. Pragmática Sociocultural, 7(3), pp. 335-369. https://doi.org/10.1515/soprag-2019-0028

6. Expósito, F., Moya, M. y Glick, P. (1998). Sexismo ambivalente: medición y correlatos. Revista de Psicología Social, 13(2), pp. 159-169. https://doi.org/10.1174/021347498760350641

7. Fiske, S., Cuddy, A. J., Glick, P. y Xu, J. (2002). A Model of (Often Mixed) Stereotype Content: Competence and Warmth Respectively Follow from Perceived Status and Competition, Journal of Personality and Social Psychology. 82(6), pp. 878-902. https://doi.org/10.1037/0022-3514.82.6.878

8. Glick, P. y Fiske, S. (1999). The Ambivalent Sexism Inventory: Differentiating Hostile and Benevolent Sexism. Journal of Personality and Social Psychology, 70(3), pp. 491-512. https://doi.org/10.1037/0022-3514.70.3.491

9. Kaul de Marlangeon, S. (2005). Descortesía de fustigación por afiliación exacerbada o refractariedad. En Bravo, D. (Ed.), Estudios de la (des)cortesía en español. Categorías conceptuales y aplicaciones a corpora orales y escritos (Vol. 1, pp. 299-318). Buenos Aires: Programa EDICE-Dunken.

10. Kaul de Marlangeon, S. (2008). Tipología del comportamiento verbal descortés en español. En Briz, A., Hidalgo, A., Albelda, M., Contreras, J. y Hernández, N., (Eds.), Cortesía y conversación: de lo escrito a lo oral. Tercer Coloquio Internacional del Programa EDICE (Vol. 3, pp. 254-266). Valencia-Estocolmo: Universidad de Valencia-Programa EDICE.

11. Kaul de Marlangeon, S. (2014). Delimitación de unidades extralingüísticas de análisis del discurso de (des)cortesía. Signo y Seña (Revista del Instituto de Lingüistica de la Universidad de Buenos Aires), 26, pp. 7-22.

12. Kaul de Marlangeon, S. (2017). Tipos de descortesía verbal y emociones en contextos de cultura hispanohablante. SOPRAG (Pragmática Sociocultural. Revista Internacional sobre Lingüistica del Español), 5(1), pp. 1-23. 
https://doi.org/10.1515/soprag-2017-0001

13. Kaul de Marlangeon, S. (2018). Fustigation impoliteness, emotions and extimacy in argentine media celebrities. Russian Journal of Linguistics, 22(1), pp. 161-174. https://doi.org/10.22363/2312-9182-2018-22-1-161-174

14. Lipovetsky, G. (1997). La tercera mujer. Barcelona: Anagrama.

15. Moya, M., Expósito, F y Padilla, J. L. (2006). Revisión de las propiedades psicométricas de las versiones larga y reducida de la Escala sobre Ideología de Género. International Journal of Clinical and Health Psychology, 6(3), pp. 709-727.

16. Wolfe, C. T. y Spencer, S. (1996). tereotypes and Prejudice: Their overt and subtle influence in the classroom. Sage Journals, 40(2), 176-185. https://doi.org/10.1177/0002764296040002008 